\title{
Biomassa, Carbono e Nitrogênio na Serapilheira Acumulada de Florestas Plantadas e Nativa
}

\author{
Verilma Barbosa ${ }^{1}$, Patrícia Barreto-Garcia ${ }^{1}$, Emanuela Gama-Rodrigues ${ }^{2}$, \\ Alessandro de Paula ${ }^{1}$
}

${ }^{1}$ Departamento de Engenharia Agrícola e Solos, Universidade Estadual do Sudoeste da Bahia - UESB, Vitória da Conquista/BA, Brasil

${ }^{2}$ Laboratório de Solos, Universidade Estadual do Norte Fluminense Darcy Ribeiro - UENF, Campos dos Goytacazes/RJ, Brasil

\begin{abstract}
RESUMO
O objetivo deste trabalho foi avaliar os estoques de biomassa, carbono e nitrogênio da serapilheira acumulada em um fragmento de Floresta Estacional Semidecidual Montana e dois plantios florestais puros (um de Pterogyne nitens e outro de Eucalyptus urophylla), no sudoeste da Bahia. As amostragens da serapilheira foram realizadas nos períodos chuvoso e seco. A biomassa da serapilheira acumulada foi superior no povoamento de eucalipto $\left(13,1 \mathrm{Mg}^{-h a^{-1}}\right)$ e inferior no povoamento de $P$. nitens $\left(1,5 \mathrm{Mg} \cdot \mathrm{ha}^{-1}\right)$, atingindo valores intermediários na floresta nativa $\left(6,3 \mathrm{Mg} \mathrm{ha}^{-1}\right)$. Essa mesma sequência foi verificada para os estoques de carbono: 5,6 Mg.ha ${ }^{-1}$ (eucalipto), 2,7 Mg.ha ${ }^{-1}$ (floresta nativa) e $0,6 \mathrm{Mg} \cdot \mathrm{ha}^{-1}$ (P. nitens). O povoamento P. nitens apresentou teores de $\mathrm{N}$ da serapilheira foliar compatíveis aos da floresta nativa. As maiores diferenças de biomassa, carbono e nitrogênio da serapilheira acumulada entre as estações chuvosa e seca foram verificadas nos povoamentos puros.
\end{abstract}

Palavras-chave: floresta estacional, Pterogyne nitens, eucalipto.

\section{Biomass, Carbon and Nitrogen in the Accumulated Litter of Planted and Native Forests}

\begin{abstract}
This study aimed to evaluate the biomass, carbon and nitrogen stocks in accumulated litter in a Semidecidual Seasonal Forest fragment and two pure forest plantations (Pterogyne nitens and Eucalyptus urophylla), located in the Southwest of Bahia. Litter samplings were carried out in the rainy and dry season. The biomass from accumulated litter was higher in eucalyptus stand $\left(13.1 \mathrm{Mg} \mathrm{ha}^{-1}\right)$ and lower in the Pterogyne nitens $\left(1.5 \mathrm{Mg} \mathrm{ha}^{-1}\right)$, reaching intermediate values in the native forest (6.3 Mg ha-1). This same sequence was checked for carbon stocks: $5.6 \mathrm{Mg} \mathrm{ha}^{-1}$ (eucalypt) $2.7 \mathrm{Mg} \mathrm{ha}^{-1}$ (native forest) and $0.6 \mathrm{Mg} \mathrm{ha}^{-1}$ (P. nitens). The P. nitens stand has $\mathrm{N}$ content of leaf's litter compatible to the native forest. The greatest differences in biomass, carbon and nitrogen of accumulated litter between the rainy and dry seasons were found in pure stands.
\end{abstract}

Keywords: seasonal forest, Pterogyne nitens, eucalypt. 


\section{INTRODUÇÃO}

O Brasil possui a segunda maior área de cobertura florestal do mundo (SFB, 2013), da qual aproximadamente 7,7 milhões de hectares são constituídos de florestas plantadas (IBÁ, 2015). Dentre as essências florestais utilizadas, destacam-se espécies exóticas, com predominância do gênero Eucalyptus, que, em 2012, já representava $76,6 \%$ da área de florestas plantadas no país (ABRAF, 2011). A utilização de espécies florestais nativas em plantios homogêneos ainda é incipiente no Brasil, o que pode ser atribuído à escassez de informações acerca de sua ecologia e silvicultura, uma vez que geralmente a escolha de espécies não considera apenas a aptidão em relação ao sítio, mas também seu potencial produtivo (Lamprecht, 1990; Tonini et al., 2005; Fraga et al., 2014).

A produtividade de um ecossistema florestal está intimamente associada à quantidade de nutrientes estocados em seus vários compartimentos, que incluem a vegetação, o solo e a serapilheira. Esse último componente refere-se à camada de matéria orgânica disposta na superfície do solo em ambientes florestais, sendo constituído por folhas, galhos, cascas, órgãos reprodutivos e detritos (Dias \& Oliveira, 1997; Costa et al., 2010).

Além de possibilitar o retorno de matéria orgânica, a serapilheira permite o reaproveitamento no ciclo de nutrientes do ecossistema, quando, por meio do processo de decomposição, libera para o solo elementos minerais que poderão ser reabsorvidos pelas raízes das plantas (Schumacher et al., 2003; Silva et al., 2007). Portanto, esse material orgânico é indispensável para a manutenção e o equilíbrio florestal de qualquer bioma.

De acordo com Delitti (1995), apesar de a ciclagem de nutrientes ser um processo comum a todos os ecossistemas florestais, as quantidades de elementos estocados nos diferentes compartimentos e os fluxos que ocorrem entre esses compartimentos são particulares a cada ecossistema. Tal padrão é resultante das características da vegetação e da ação de variáveis climáticas, que podem alterar a quantidade de serapilheira aportada em determinado sítio, bem como favorecer ou não sua decomposição.

Dessa forma, o estudo dos estoques de biomassa e nutrientes da serapilheira acumulada em povoamentos florestais, em diferentes estações do ano, pode evidenciar a capacidade de o sistema reservar ou repor nutrientes. Além disso, estudos dessa natureza contribuem para a avaliação da influência do regime hídrico como agente regulador do processo de produção e acúmulo de serapilheira, contribuindo para o entendimento da estrutura e funcionamento desses ecossistemas.

$\mathrm{Na}$ Bahia, em particular na região sudoeste do estado, ainda são escassas as pesquisas relacionadas à dinâmica de deposição e ao acúmulo de serapilheira em ecossistemas florestais nativos e plantios florestais de espécies exóticas ou nativas. Dentre as espécies nativas que apresentam potencial para utilização no estado, Pterogyne nitens Tul. destaca-se por se adaptar às condições ambientais da região, possuir rápido crescimento e gerar madeira de valor econômico para diferentes finalidades (Lorenzi, 1992). Além disso, a referida espécie apresenta a capacidade de se estabelecer em solos pobres em nutrientes e matéria orgânica, como consequência da capacidade de fixar nitrogênio pela simbiose com bactérias diazotróficas e fungos micorrízicos (Santos et al., 2008).

Diante disso, o objetivo deste trabalho foi avaliar os estoques de biomassa, carbono e nitrogênio da serapilheira acumulada em três ecossistemas florestais (um fragmento de Floresta Estacional Semidecidual Montana e dois plantios florestais puros, um de $P$. nitens e outro de Eucalyptus urophylla S. T. Blake).

\section{MATERIAL E MÉTODOS}

O estudo foi conduzido em três áreas, sendo uma de floresta nativa e duas de plantios florestais homogêneos, um de P. nitens Tul. e outro de eucalipto (E. urophylla S. T. Blake), com seis e cinco anos de idade, respectivamente, localizados na região Sudoeste da Bahia, no município de Vitória da Conquista.

A vegetação do fragmento de floresta nativa é classificada como Floresta Estacional Semidecidual Montana, conhecida regionalmente como mata de cipó. É constituída de mesofanerófitos (plantas lenhosas de porte médio, com gemas aéreas situadas acima de $0,25 \mathrm{~m}$ do solo e protegidas por folhas reduzidas, com altura variando entre 10 e $20 \mathrm{~m}$ ), parcialmente caducifólios e envolvidos por lianas, com predominância de ecotipos da família Leguminosae, destacando-se o gênero Parapiptadenia (Veloso et al., 1991; IBGE, 2012). 
Os povoamentos de $P$. nitens e eucalipto foram estabelecidos em espaçamento $3 \times 3 \mathrm{~m}$, com adubação localizada na cova, composta de $100 \mathrm{~g}$ de superfosfato simples. O preparo do solo foi realizado com o uso de gradagem e abertura de covas. O povoamento de P. nitens apresentava árvores com diâmetro à altura de $1,3 \mathrm{~m}$ (DAP), variando de 2,9 a $13,5 \mathrm{~cm}$, altura entre 6,1 e 10,5 m e incremento médio anual (IMA) de $10,1 \mathrm{~m}^{3} \cdot \mathrm{ha}^{-1} \cdot \mathrm{ano}^{-1}$. O povoamento de eucalipto apresentava DAP médio de $16,0 \mathrm{~cm}$, altura média de 16,7 m e IMA de $29,9 \mathrm{~m}^{3} \cdot \mathrm{ha}^{-1} \cdot$ ano $^{-1}$ (Santos et al., 2015).

A região tem relevo plano a levemente ondulado e altitude média de $840 \mathrm{~m}$. O clima é do tipo Cwb, segundo classificação de Köppen, tropical de altitude, apresentando média anual de temperatura de $25^{\circ} \mathrm{Ce}$ precipitação de $850 \mathrm{~mm}$. Os solos das áreas estudadas possuem textura argiloarenosa e pertencem a uma mesma classe: Latossolo Amarelo Distrófico (Santos et al., 2006). A Tabela 1 apresenta a granulometria e a caracterização química desses solos na profundidade de 0 a $5 \mathrm{~cm}$.

As coletas de serapilheira acumulada foram realizadas entre novembro de 2010 (período chuvoso) e maio de 2011 (período seco). Para isso, em cada uma das áreas estudadas, foram demarcadas quatro parcelas de $21 \times 21 \mathrm{~m}$. As amostras de serapilheira foram retiradas com o auxílio de uma moldura de madeira, de forma quadrada, medindo $0,5 \mathrm{~m}$ x 0,5 $\mathrm{m}\left(0,25 \mathrm{~m}^{2}\right)$. Em cada parcela, a moldura foi lançada de forma aleatória sobre o solo com três repetições, que foram reunidas para formar uma amostra composta.

O material circunscrito à moldura foi cuidadosamente retirado, evitando a coleta de solo e raízes vivas. Após cada coleta, os materiais amostrados foram secos ao ar e triados, separando-se cinco frações (folhas, galhos, casca, estruturas reprodutivas e material amorfo) e retirando-se as impurezas. Posteriormente, as frações foram secas em estufa de ventilação forçada a $60^{\circ} \mathrm{C}$ por 72 horas e pesadas em balança de precisão $(0,01 \mathrm{~g})$. Os resultados de massa seca $(\mathrm{g})$ obtidos foram convertidos em kg.ha ${ }^{-1}$.

As amostras secas de serapilheira foram moídas e submetidas a análises químicas para determinar os teores de $\mathrm{N}$ total pelo método Kjeldahl, descrito por Bataglia et al. (1983), e de C, por oxidação com $\mathrm{K}_{2} \mathrm{Cr}_{2} \mathrm{O}_{7}$ 0,4 mol.L $\mathrm{L}^{-1}$ em meio ácido (Anderson \& Ingram, 1996).

Para comparar a biomassa da serapilheira acumulada (das frações e total) e seus teores e estoques de carbono e nitrogênio entre os ecossistemas florestais, bem como entre as estações, foi realizado o teste $t$ de Student a 5\% de significância, empregando-se o programa estatístico SISVAR ${ }^{\circ}$.

\section{RESULTADOS E DISCUSSÃO}

Considerando as médias das duas estações do ano, a maior quantidade de serapilheira total acumulada sobre o solo foi observada no eucalipto $\left(13,1 \mathrm{Mg} \cdot \mathrm{ha}^{-1}\right)$, seguido da floresta nativa $\left(6,3 \mathrm{Mg}^{-} \mathrm{ha}^{-1}\right)$ e do povoamento de P. nitens (1,5 Mg.ha $\left.{ }^{-1}\right)$. Obedecendo a essa mesma sequência, os estoques totais de carbono orgânico da serapilheira foram de 5,6 $\mathrm{Mg}^{-} \mathrm{ha}^{-1}$, para o eucalipto, 2,7 Mg.ha- ${ }^{-1}$, para a floresta nativa, e $0,6 \mathrm{Mg} \cdot \mathrm{ha}^{-1}$, para P. nitens, o que corresponde a teores médios de $\mathrm{C}$ de $43 \%$, $40 \%$ e $27 \%$, respectivamente (Tabela 2).

Resultados superiores aos observados para o eucalipto foram verificados por Barreto et al. (2008) que, ao estudar plantações de Eucalyptus grandis $x$ Eucalyptus urophylla com cinco anos de idade, estimaram a serapilheira em

Tabela 1. Caracterização granulométrica e química do solo (profundidade de 0 a $5 \mathrm{~cm}$ ) sob três ecossistemas florestais no sudoeste da Bahia.

Table 1. Granulometric and chemical composition of soils (0-5 cm depth) under three forest ecosystems in the Southwest of Bahia.

\begin{tabular}{|c|c|c|c|c|c|c|c|c|c|c|}
\hline \multirow{2}{*}{ Ecossistema } & Argila & Areia & Silte & \multirow{2}{*}{ pH } & MO & $\mathbf{H}+\mathbf{A l}$ & $\mathbf{P}$ & $\mathbf{K}$ & $\mathrm{Ca}$ & Mg \\
\hline & \multicolumn{3}{|c|}{$----\mathrm{g} \cdot \mathrm{kg}^{-1}-\ldots-$} & & $\mathrm{g} \cdot \mathrm{dm}^{-3}$ & \multicolumn{5}{|c|}{---ー-— cmolc. $\mathrm{dm}^{3}------$} \\
\hline Floresta nativa & 450 & 540 & 10 & 4,3 & 46,0 & 10,1 & 2,0 & 0,12 & 1,20 & 0,78 \\
\hline Eucalyptus urophylla & 490 & 500 & 10 & 4,8 & 26,3 & 5,8 & 2,0 & 0,08 & 0,63 & 0,58 \\
\hline Pterogyne nitens & 380 & 608 & 12 & 5,6 & 16,3 & 2,6 & 1,3 & 0,20 & 1,13 & 1,00 \\
\hline
\end{tabular}

Análises realizadas de acordo com EMBRAPA (1979): granulometria pelo método da pipeta; pH (água); P e K extraíveis por Mehlich-1; Ca, Mg e Al trocáveis por $\mathrm{KCl} 1 \mathrm{~mol} \mathrm{L-1}$ e matéria orgânica (MO) por oxidação com $\mathrm{Na}_{2} \mathrm{Cr}_{2} \mathrm{O}_{7} 4 \mathrm{~N}$. Para cada ecossistema florestal, foram utilizadas quatro amostras compostas de 20 amostras simples, coletadas nas mesmas parcelas utilizadas para a coleta da serapilheira. 
Tabela 2. Valores médios de biomassa, teores e estoques de carbono e nitrogênio e relação $\mathrm{C} / \mathrm{N}$ das frações da serapilheira de três ecossistemas florestais no sudoeste da Bahia.

Table 2. Average biomass values, concentration and carbon and nitrogen stocks and $C / N$ ratio of the litter's fractions from three forest ecosystems in southwest Bahia.

\begin{tabular}{|c|c|c|c|c|c|c|}
\hline \multirow{2}{*}{ Ecossistema } & Folhas & Galhos & Cascas & $\mathbf{E R}^{1}$ & MA & Total \\
\hline & \multicolumn{6}{|c|}{ Biomassa $\left(\mathrm{Mg} \cdot \mathrm{ha}^{-1}\right)$} \\
\hline Floresta nativa & $2,52 \mathrm{~b}$ & $2,76 \mathrm{a}$ & $0,64 \mathrm{a}$ & $0,22 \mathrm{~b}$ & $0,11 \mathrm{a}$ & $6,24 \mathrm{~b}$ \\
\hline Eucalyptus urophylla & $8,44 \mathrm{a}$ & $3,54 \mathrm{a}$ & $0,25 \mathrm{~b}$ & $0,65 \mathrm{a}$ & $0,19 \mathrm{a}$ & $13,07 \mathrm{a}$ \\
\hline \multirow[t]{2}{*}{ Pterogyne nitens } & $0,46 \mathrm{c}$ & $1,00 \mathrm{~b}$ & $0,01 \mathrm{c}$ & $0,02 \mathrm{c}$ & $0,02 \mathrm{~b}$ & $1,49 \mathrm{c}$ \\
\hline & \multicolumn{6}{|c|}{ Carbono (\%) } \\
\hline Floresta nativa & $43,11 \mathrm{a}$ & $43,20 \mathrm{a}$ & $42,90 \mathrm{a}$ & $45,00 \mathrm{a}$ & $39,00 \mathrm{a}$ & \\
\hline Eucalyptus urophylla & $41,90 \mathrm{a}$ & $45,30 \mathrm{a}$ & $46,20 \mathrm{a}$ & $43,30 \mathrm{a}$ & $22,50 \mathrm{~b}$ & \\
\hline \multirow{2}{*}{ Pterogyne nitens } & $43,50 \mathrm{a}$ & $43,50 \mathrm{a}$ & $15,20 \mathrm{~b}$ & $28,70 \mathrm{~b}$ & $4,30 \mathrm{c}$ & \\
\hline & \multicolumn{6}{|c|}{ Carbono $\left(\mathrm{Mg} \mathrm{ha}^{-1}\right)$} \\
\hline Floresta nativa & $1,09 \mathrm{~b}$ & $1,19 a$ & $0,28 \mathrm{a}$ & $0,10 \mathrm{~b}$ & $0,04 \mathrm{a}$ & $2,69 \mathrm{~b}$ \\
\hline Eucalyptus urophylla & $3,54 \mathrm{a}$ & $1,60 \mathrm{a}$ & $0,11 \mathrm{~b}$ & $0,28 \mathrm{a}$ & $0,04 \mathrm{a}$ & $5,58 \mathrm{a}$ \\
\hline \multirow[t]{2}{*}{ Pterogyne nitens } & $0,20 \mathrm{c}$ & $0,43 \mathrm{~b}$ & $0,00 \mathrm{c}$ & $0,01 \mathrm{c}$ & $0,00 \mathrm{~b}$ & $0,64 \mathrm{c}$ \\
\hline & \multicolumn{6}{|c|}{ Nitrogênio (\%) } \\
\hline Floresta nativa & $1,9 \mathrm{a}$ & $1,0 \mathrm{a}$ & $1,3 \mathrm{a}$ & $1,4 \mathrm{a}$ & $1,1 \mathrm{a}$ & \\
\hline Eucalyptus urophylla & $0,9 \mathrm{~b}$ & $0,2 \mathrm{~b}$ & $0,4 \mathrm{~b}$ & $0,6 \mathrm{~b}$ & $0,5 \mathrm{~b}$ & \\
\hline \multirow[t]{2}{*}{ Pterogyne nitens } & $2,2 \mathrm{a}$ & $0,8 \mathrm{a}$ & $0,2 \mathrm{~b}$ & $1,3 \mathrm{a}$ & $0,7 \mathrm{ab}$ & \\
\hline & \multicolumn{6}{|c|}{ Nitrogênio $\left(\mathrm{kg} \cdot \mathrm{ha}^{-1}\right)$} \\
\hline Floresta nativa & $46,6 \mathrm{~b}$ & $28,4 \mathrm{a}$ & $8,4 \mathrm{a}$ & $3,0 \mathrm{a}$ & $1,2 \mathrm{a}$ & $87,6 \mathrm{a}$ \\
\hline Eucalyptus urophylla & $71,7 \mathrm{a}$ & $6,0 \mathrm{~b}$ & $0,9 \mathrm{~b}$ & $3,9 \mathrm{a}$ & $1,0 \mathrm{a}$ & 83,6 a \\
\hline \multirow[t]{2}{*}{ Pterogyne nitens } & $10,0 \mathrm{c}$ & $8,2 \mathrm{~b}$ & $0,0 \mathrm{~b}$ & $0,2 \mathrm{~b}$ & $0,0 \mathrm{~b}$ & $18,4 \mathrm{~b}$ \\
\hline & \multicolumn{6}{|c|}{ Relação C/N } \\
\hline Floresta nativa & $23,3 \mathrm{~b}$ & $41,9 \mathrm{~b}$ & $32,7 \mathrm{~b}$ & $32,6 \mathrm{~b}$ & $34,8 \mathrm{~b}$ & $30,7 \mathrm{~b}$ \\
\hline Eucalyptus urophylla & $49,3 \mathrm{a}$ & $266,5 \mathrm{a}$ & 124,9 a & $71,0 \mathrm{a}$ & $45,0 \mathrm{~b}$ & $66,7 \mathrm{a}$ \\
\hline Pterogyne nitens & $20,0 \mathrm{~b}$ & $53,0 \mathrm{~b}$ & $95,0 \mathrm{a}$ & $21,9 \mathrm{~b}$ & $61,4 \mathrm{a}$ & $34,8 \mathrm{~b}$ \\
\hline
\end{tabular}

${ }^{1}$ ER: estruturas reprodutivas; MA: material amorfo. Valores apresentados são provenientes da média de quatro repetições. Médias seguidas da mesma letra na coluna não diferem entre si pelo teste t a $5 \%$ de significância.

15,3 Mg.ha-1, com teor médio de carbono orgânico de 40,4\%. Para a mesma espécie, Cunha et al. (2013) obtiveram biomassa inferior em povoamento com quatro

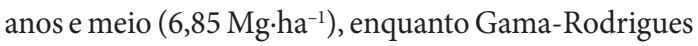
\& Barros (2002) encontraram resultado próximo em um povoamento com dez anos de idade $\left(13,5 \mathrm{Mg} \cdot \mathrm{ha}^{-1}\right)$.

As quantidades de biomassa e carbono orgânico encontradas na serapilheira da floresta nativa aproximaram-se dos valores quantificados por Godinho et al. (2014) em um trecho de Floresta Estacional Semidecidual Submontana, no estado do Espírito Santo, tendo tais autores observado 5,5 ${\mathrm{Mg} \cdot h a^{-1}}^{-1}$ de biomassa e 2,8 Mg.ha-1 de carbono orgânico.

$\mathrm{O}$ acúmulo de serapilheira é regulado pela quantidade de material decíduo aportado sobre o piso florestal e, ao mesmo tempo, por sua taxa de decomposição (Haag, 1985). Por sua vez, de acordo com O'Connell \& Sankaran (1997), o aporte e a decomposição da serapilheira são influenciados pela cobertura florestal, que determina a natureza do material formador da serapilheira e a população de organismos decompositores, e pelas condições do ambiente (como disponibilidade de água e temperatura). Além disso, o processo de decomposição pode sofrer interferências das propriedades físico-químicas do solo.

Como as condições de solo e clima das três áreas estudadas são semelhantes, é provável que o maior acúmulo de material decíduo verificado no povoamento de eucalipto esteja relacionado à quantidade e à qualidade dos resíduos depositados pela espécie ou, ainda, às características da população decompositora. Nesse sentido, os menores teores de nitrogênio e maiores relações $\mathrm{C} / \mathrm{N}$ encontradas na serapilheira desse ecossistema (Tabela 2), em relação à floresta nativa e ao povoamento de $P$. nitens, corroboram essa hipótese, uma vez que denota maior recalcitrância do material. 
Fonseca et al. (1993) também observaram maior quantidade de serapilheira em povoamentos de Eucalyptus citriodora, quando comparados a uma área de floresta nativa, atribuindo o resultado à maior relação $\mathrm{C} / \mathrm{N}$ dos resíduos depositados pela espécie. $\mathrm{Na}$ literatura são comumente relatadas baixas taxas de decomposição em plantações de eucalipto, como efeito da baixa qualidade nutricional e orgânica da serapilheira (Adams \& Atiwill, 1986; Costa et al., 2005). Todavia, de acordo com Gama-Rodrigues et al. (1999), embora o maior acúmulo de serapilheira indique baixa taxa de ciclagem de nutrientes, não significa necessariamente menor capacidade de melhorar a fertilidade do solo.

Por outro lado, o reduzido acúmulo de serapilheira observado no povoamento de $P$. nitens pode representar elevada velocidade de decomposição, uma vez que essa cobertura florestal, em geral, apresentou os maiores teores de nitrogênio $(\mathrm{N})$ das frações da serapilheira, embora estatisticamente similares aos da floresta nativa (Tabela 2). Tal semelhança pode ser justificada pela capacidade de fixar nitrogênio da espécie $P$. nitens e, ao mesmo tempo, pela ocorrência de espécies com a mesma capacidade na floresta nativa. De acordo com Constantinides \& Fownes (1994), maiores concentrações de nitrogênio e, consequentemente, maiores taxas de decomposição são esperadas para espécies que possuem potencial para fixação de $\mathrm{N}$, como é o caso daquelas da família Fabaceae.

Dessa forma, é provável que o valor intermediário de acúmulo total de serapilheira da floresta nativa $\left(6,3 \mathrm{Mg} \cdot \mathrm{ha}^{-1}\right)$, com proporção de folhas semelhante à dos galhos (Tabela 2), esteja relacionado a características do ecossistema, que apresenta maior diversidade de espécies, predominância das famílias Leguminosae e Fabaceae e um significativo número de indivíduos com DAP superior a $5,0 \mathrm{~cm}$ (Soares, 2012). Isso propicia um ambiente biologicamente mais complexo do que o existente nos povoamentos puros, o que deve refletir em maior quantidade e diversidade dos materiais decíduos depositados sobre o solo. Como consequência, também proporciona maior heterogeneidade da comunidade de organismos decompositores, podendo degradar mais rapidamente os materiais menos lignificados como as folhas.

Segundo essa perspectiva, Gama-Rodrigues \& Barros (2002) avaliaram a decomposição do folhedo de Eucalyptus grandis $\mathrm{x}$ E. urophylla no ambiente de um fragmento de floresta natural e no próprio ambiente do povoamento da espécie e observaram maior perda de massa no ambiente natural $(52,5 \%)$ em relação ao plantio de eucalipto $(42,6 \%)$, o que evidencia que o processo de decomposição é também influenciado pela qualidade do ambiente, principalmente por aqueles fatores que promovem mais atividade microbiana.

A fração folhas foi o principal material formador da serapilheira no povoamento de eucalipto (65\%), enquanto na floresta nativa e $P$. nitens foram os galhos que representaram maior proporção no acúmulo total (44\% e 67\%, respectivamente) (Tabela 2). O povoamento de $P$. nitens acumulou $30,7 \%$ de folhas, o menor percentual dessa fração verificado no estudo (Tabela 2), evidenciando alta taxa de decomposição do material foliar. Entretanto, esse ecossistema apresentou o maior acúmulo de galhos (66,7\%), que são materiais normalmente lenhosos, mais resistentes à decomposição do que as folhas e ramos tenros ainda não lignificados. Assim, o acúmulo superior de galhos está relacionado com a composição química e orgânica desse material, uma vez que, dentro de uma mesma região climática, este é o fator determinante das taxas de decomposição (Swift et al., 1979).

$\mathrm{Na}$ floresta nativa, a participação média das frações após os galhos, que representaram $44,1 \%$ da serapilheira, ocorreu na seguinte ordem: folhas $(40,4 \%)>$ cascas $(10,3 \%)>$ estruturas reprodutivas $(3,5 \%)>$ material amorfo $(1,7 \%)$.

Em todos os três ecossistemas, a fração folhas representou o principal contribuinte de nitrogênio da serapilheira, tanto ao se considerar os teores quanto os estoques desse elemento (Tabela 2). Tais resultados são corroborados pelos observados por Meguro et al. (1979), Vital et al. (2004) e Godinho et al. (2014), que relataram que a folha foi o componente da serapilheira que possuía quantidade mais significativa de nitrogênio e outros nutrientes, constituindo uma importante via de retorno dos elementos minerais da vegetação para o solo.

Apesar de o povoamento de $P$. nitens ter apresentado, em geral, os maiores teores de nitrogênio nas frações da serapilheira, foi o ecossistema florestal que obteve menor quantidade desse elemento estocada na serapilheira total $\left(18,4 \mathrm{~kg} \cdot \mathrm{ha}^{-1}\right)$, como resultado do menor acúmulo de biomassa, provavelmente decorrente de maior taxa de decomposição. Tais características indicam 
que a espécie apresenta potencial para ser utilizada em programas de recuperação de áreas degradadas.

A floresta nativa e o eucalipto apresentaram estoques de $\mathrm{N}$ semelhantes entre si (em média, a 85,6 kg.ha-1). Resultado próximo foi encontrado por Godinho et al. (2014) em Floresta Estacional Semidecidual Submontana $\left(94,9 \mathrm{~kg} \cdot \mathrm{ha}^{-1}\right)$.

A maior diferença de acúmulo entre as estações chuvosa e seca ocorreu no povoamento de $P$. nitens, que oscilou de 1,9 para 1,1 $\mathrm{Mg} \cdot \mathrm{ha}^{-1}$, representando redução de $41 \%$ (Tabela 3 ). Essa mesma tendência de diminuição, embora em menor proporção, ocorreu na floresta nativa (-7\%) (Tabela 3). Esse comportamento pode ser indicativo de maior adaptabilidade das espécies dos dois povoamentos, que propiciou menor perda de biomassa foliar no período de déficit hídrico. Outra possível explicação estaria relacionada à influência mais efetiva e rápida de variáveis climáticas, que se refletiu em maior produção e acúmulo de serapilheira ainda no período chuvoso. Maior deposição de serapilheira logo após a estação chuvosa tem sido notada em diferentes ecossistemas florestais (Froufe, 1999; Santana et al., 2009; Lima et al., 2010).

O único povoamento que mostrou maior acúmulo de serapilheira no período seco foi o de eucalipto $\left(14,9 \mathrm{Mg} \mathrm{ha}^{-1}\right)$, superando o período chuvoso $\left(11,2 \mathrm{Mg} \mathrm{ha}^{-1}\right)$ em mais de $30 \%$ (Tabela 3 ), o que pode ser resultante da perda significativa de folhas no período de maior restrição hídrica. No entanto, Kolm \& Pogiane (2003) observaram, em um povoamento de Eucalyptus grandis, maior deposição de serapilheira na estação chuvosa. Ao estudarem acúmulo de serapilheira em plantios florestais puros, Santana et al. (2009) observaram que a maior diferença encontrada entre as estações ocorreu no plantio de Mimosa caesalpiniifolia, que apresentou biomassa $142 \%$ superior no período seco, e atribuíram o resultado à possível característica de deciduidade da espécie.

$\mathrm{Na}$ floresta nativa, os estoques de carbono e nitrogênio da serapilheira total pouco variaram entre as estações, representando diferenças de apenas $4 \%$ e $2 \%$, respectivamente. Para o eucalipto, os estoques de carbono foram maiores na estação seca, superando em $52 \%$ a estação chuvosa. Já no povoamento de $P$. nitens, verificou-se diminuição do conteúdo de carbono na estação seca, que representou redução de 34\%. Em todos os ecossistemas estudados, as variações nos estoques de $\mathrm{C}$ entre as estações climáticas foram um reflexo das diferenças na biomassa da serapilheira. Ao avaliarem a serapilheira acumulada em um fragmento de Mata Atlântica, Borém \& Ramos (2002) não verificaram diferenças significativas dos conteúdos de carbono entre as estações. Já Silva et al. (2009), ao estudarem a floresta de transição Amazônia/Cerrado no Norte do Mato Grosso, observaram diminuição da concentração de carbono da fração foliar da serapilheira no período seco.

Tabela 3. Estoques de biomassa, carbono e nitrogênio da serapilheira acumulada sobre o solo em três ecossistemas florestais nas estações seca e chuvosa.

Table 3. Biomass, carbon and nitrogen stocks from soil's accumulated litter in three forest ecosystems in Dry and Rainy Seasons.

\begin{tabular}{|c|c|c|c|}
\hline \multirow{3}{*}{ Ecossistema } & Chuvosa & Seca & Chuvosa/seca $\times 100$ \\
\hline & \multicolumn{2}{|c|}{ - - Mg.ha ${ }^{-1}----$} & --- $\%----$ \\
\hline & \multicolumn{3}{|c|}{ Biomassa } \\
\hline Floresta nativa & $6,47 \mathrm{Ab}$ & $6,02 \mathrm{Ab}$ & $-7,0$ \\
\hline Eucalyptus urophylla & $11,24 \mathrm{Ba}$ & $14,90 \mathrm{Aa}$ & 32,6 \\
\hline \multirow[t]{2}{*}{ Pterogyne nitens } & $1,88 \mathrm{Ac}$ & $1,11 \mathrm{Bc}$ & $-40,9$ \\
\hline & \multicolumn{3}{|c|}{ Carbono } \\
\hline Floresta nativa & $2,62 \mathrm{Ab}$ & $2,73 \mathrm{Ab}$ & 4,2 \\
\hline Eucalyptus urophylla & $4,47 \mathrm{Ba}$ & $6,80 \mathrm{Aa}$ & 52,0 \\
\hline \multirow[t]{2}{*}{ Pterogyne nitens } & $0,75 \mathrm{Ac}$ & $0,50 \mathrm{Bc}$ & $-33,9$ \\
\hline & \multicolumn{3}{|c|}{ Nitrogênio } \\
\hline Floresta nativa & $0,08 \mathrm{Aa}$ & $0,09 \mathrm{Aa}$ & 2,0 \\
\hline Eucalyptus urophylla & $0,09 \mathrm{Aa}$ & 0,08 Bab & $-14,4$ \\
\hline Pterogyne nitens & $0,02 \mathrm{Ab}$ & $0,02 \mathrm{Bb}$ & $-32,6$ \\
\hline
\end{tabular}

Valores apresentados são provenientes da média de quatro repetições. Médias seguidas da mesma letra maiúscula na linha e minúscula na coluna não diferem entre si pelo teste t a $5 \%$ de significância; $\mathrm{n}=4$. 
Nos povoamentos puros, os estoques de nitrogênio foram maiores na estação chuvosa, superando o período seco em $14 \%$ (eucalipto) e 33\% (P. nitens) (Tabela 3). Além da queda e decomposição de serapilheira, as chuvas representam a principal fonte de nitrogênio para o sistema solo-planta (Luizão, 1989), o que pode explicar o aumento do conteúdo de $\mathrm{N}$ observado no período chuvoso.

Em geral, verifica-se que a quantidade de biomassa, carbono e nitrogênio que retorna ao solo por meio da serapilheira acumulada foi influenciada pela época do ano, o que foi constatado por outros estudos (Attiwill et al., 1978; Borém \& Ramos, 2002; Godinho et al., 2014). Diversos outros fatores também condicionam esse retorno, dentre eles pode-se citar a diversidade de espécies e a idade do povoamento (O'Connell et al., 1978), a composição química dos resíduos (Tian et al., 1992), a atuação dos organismos edáficos (Melillo et al., 1982) e as propriedades físico-químicas do solo.

\section{CONCLUSÕES}

A biomassa da serapilheira acumulada foi superior

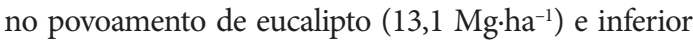
no povoamento de $P$. nitens $\left(1,5 \mathrm{Mg} \cdot \mathrm{ha}^{-1}\right)$, atingindo valores intermediários na floresta nativa $\left(6,3 \mathrm{Mg} \cdot \mathrm{ha}^{-1}\right)$. Essa mesma sequência foi verificada para os estoques de

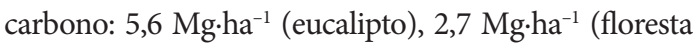
nativa) e $0,6 \mathrm{Mg} \cdot \mathrm{ha}^{-1}$ (P. nitens).

O povoamento de $P$. nitens apresenta teores de $\mathrm{N}$ da serapilheira foliar compatíveis aos da floresta nativa e inferiores aos do povoamento de eucalipto, o que denota potencial de utilização da espécie $P$. nitens para recuperação de áreas degradadas.

$\mathrm{Na}$ composição da serapilheira total, as frações que apresentam maior contribuição são folhas, para o eucalipto, e galhos, para floresta nativa e $P$. nitens.

As maiores diferenças de biomassa, carbono e nitrogênio da serapilheira acumulada entre as estações chuvosa e seca foram verificadas nos povoamentos puros. Os maiores estoques de biomassa foram observados na estação chuvosa, para os povoamentos de P. nitens e de floresta nativa, e na estação seca, para o povoamento de eucalipto.

\section{STATUS DA SUBMISSÃO}

Recebido: 21 jul., 2016

Aceito: 01 out., 2016

\section{AUTOR(ES) PARA CORRESPONDÊNCIA}

\section{Patrícia Barreto-Garcia}

Departamento de Engenharia Agrícola e Solos, Universidade Estadual do Sudoeste da Bahia UESB, Estrada do Bem Querer, Km 4, CP 95, CEP 45031-300, Vitória da Conquista, BA, Brasil e-mail: patriciabarreto@uesb.edu.br

\section{REFERÊNCIAS}

Adams MA, Atiwill PM. Nutrient cycling an nitrogen mineralization in eucalypt forests south-easthern Australia. I- Nutrient cycling and nitrogen turnover. Plant and Soil 1986; 92: 319-339. http://dx.doi.org/10.1007/BF02372482.

Anderson JD, Ingram JSI. Tropical soil biology and fertility: a handbook of methods. 2nd ed. Wallingford: CAB International; 1996.

Associação Brasileira de Produtores de Florestas Plantadas - ABRAF. Anuário estatístico da ABRAF 2011 ano base 2010. Brasília: ABRAF; 2011 [citado em 2013 jul. 05]. 130 p. Disponível em: http://www.abraflor.org.br/estatisticas/ ABRAF11/ABRAF11-EN.pdf.

Attiwill PM, Guthrie HB, Leuning R. Nutrient cycling in a E. obliqua (L'Hérit) forest. I - Litter production and nutrient return. Australian Journal of Botany 1978; 26(1): 79-91. http://dx.doi.org/10.1071/BT9780079.

Barreto PAB, Gama-Rodrigues EF, Gama-Rodrigues AC, Barros NF, Fonseca S. Atividade, carbono e nitrogênio da biomassa microbiana em plantações de eucalipto, em uma seqüência de idades. Revista Brasileira de Ciência do Solo 2008; 32: 611-619.

Bataglia OC, Furlani AMC, Teixeira JPF, Furlani PR, Gallo JR. Métodos de análise química de plantas. Campinas: Instituto Agronômico; 1983. 48 p. Boletim Técnico, 78.

Borém RAT, Ramos DP. Variação estacional e topográfica de nutrientes na serapilheira de um fragmento de mata atlântica. Cerne. 2002; 8(2): 42-59.

Constantinides M, Fownes JH. Nitrogen mineralization from leaves and litter of tropical plants: relationship to nitrogen, lignin and soluble polyphenol concentrations. Soil Biology \& Biochemistry 1994; 26(1): 49-55. http:// dx.doi.org/10.1016/0038-0717(94)90194-5.

Costa CCA, Camacho RGV, Macedo ID, Silva PCM. Análise comparativa da produção de serapilheira em fragmentos arbóreos e arbustivos em área de caatinga na FLONA de Açu-RN. Revista Árvore 2010; 34(2): 259-265.

Costa GS, Gama-Rodrigues AC, Cunha GM. Decomposição e liberação de nutrientes da serapilheira foliar em povoamentos de Eucalyptus grandis no Norte Fluminense. Revista Árvore 2005; 29(4): 563-570. 
Cunha FV No, Leles PSS, Pereira MG, Bellumath VGH, Alonso JM. Acúmulo e decomposição da serapilheira em quatro formações florestais. Revista Ciência Florestal 2013; 23(3): 379-387.

Delitti WBC. Estudos de ciclagem de nutrientes: instrumentos para análise funcional de ecossistemas terrestres. Oecologia Brasiliensis: 1995; 1: 469-486.

Dias HCT, Oliveira AT. Variação temporal e espacial da produção de serrapilheira em uma área de Floresta Estacional Semidecídua Montana em Lavras-MG. Revista Árvore 1997; 21: 11-26.

Empresa Brasileira de Pesquisa Agropecuária - EMBRAPA. Serviço Nacional de Levantamento e Classificação de Solos. Manual de métodos de análise de solo. Rio de Janeiro: Embrapa-SNLCS; 1979. 255 p

Fonseca S, Barros NF, Novais RF, Costa LM, Leal PGL, Neves JCL. Alterações em um latossolo sob eucalipto, mata natural e pastagem. I. Propriedades físicas e químicas. Revista Árvore 1993; 17(3): 271-288.

Fraga MP, Barreto PAB, Paula A. Estimação de volume de Pterogyne nitens em plantio puro no sudoeste da Bahia. Pesquisa Florestal Brasileira 2014; 34(79): 207-215. http:// dx.doi.org/10.4336/2014.pfb.34.79.703.

Froufe LCM. Decomposição de serrapilheira e aporte de nutrientes em plantios puros e consorciados de Eucalyptus grandis Maiden, Pseudosamanea guachapele Dugand e Acacia mangium Wild [dissertação]. Seropédica: Universidade Federal Rural do Rio de Janeiro; 1999.

Gama-Rodrigues AC, Barros NF, Mendonça ES. Alterações edáficas sob plantios puros e misto de espécies florestais no Sudeste da Bahia. Revista Brasileira de Ciência do Solo 1999; 23: 581-592.

Gama-Rodrigues AC, Barros NF. Ciclagem denutrientes em floresta natural e em plantios de eucalipto e de dandá no sudeste da Bahia, Brasil. Revista Árvore 2002; 26(2): 193-207.

Godinho TO, Caldeira MVW, Rocha JHT, Caliman JP, Trazzi PA. Quantificação de biomassa e nutrientes na serapilheira acumulada em trecho de Floresta Estacional Semidecidual Submontana, ES. Cerne 2014; 20(1): 11-20.

Haag HP. Ciclagem de nutriente em florestas tropicais. Campinas: Fundação Cargill; 1985. 114 p.

Indústria Brasileira de produtores de Árvores - IBÁ. Relatório IBÁ 2015: ano base 2014. Brasília; 2015. 64 p.

Instituto Brasileiro de Geografia e Estatística - IBGE. Manual técnico da vegetação brasileira. 2. ed. Rio de Janeiro; 2012. 271 p.

Kolm L, Pogiane F. Ciclagem de nutrientes em povoamentos de Eucalyptus grandis submetidos à prática de desbastes progressivos. Scientia Forestalis 2003; 63: 79-93.

Lamprecht H. Silvicultura nos trópicos: ecossistemas florestais e respectivas espécies arbóreas: possibilidades e métodos de aproveitamento sustentado. Eschborn Deutsche Gesellschaft für Technische Zusammenarbeit Rossdorf; 1990. 343 p.

Lima SS, Leite LFC, Aquino AM, Oliveira FC, Castro AJF. Serapilheira e teores de nutrientes em argilossolo sob diferentes manejos no Norte do Piauí. Revista Árvore 2010; 34(1): 75-84.

Lorenzi H. Árvores brasileiras: manual de identificação e cultivo de plantas arbóreas nativas do Brasil. Nova Odessa: Editora Plantarum; 1992. 368 p.

Luizão FJ. Litter production and mineral element input to the forest floor in a central Amazonian forest. GeoJournal 1989; 19(4): 407-417. http://dx.doi.org/10.1007/BF00176910.

Meguro M, Vinueza GN, Delitti WBC. Ciclagem de nutrientes minerais na mata mesófila secundária - São Paulo. I - Produção e conteúdo de nutrientes minerais no folhedo. Boletim de Botânica, São Paulo 1979; 7(0): 11-31. http://dx.doi.org/10.11606/issn.2316-9052.v7i0p11-31.

Melillo JM, Aber JD, Muratore JF. Nitrogen and lignin control of hardwood leaf litter decomposition dynamics. Ecology 1982; 63(3): 621-626. http://dx.doi.org/10.2307/1936780.

O'Connell AM, Grove TS, Dimmock GM. Nutrients in the litter on Jarrah forest soils. Australian Journal of Ecology, Collingwood 1978; 3(3): 253-260. http://dx.doi. org/10.1111/j.1442-9993.1978.tb01175.x.

O’Connell AM, Sankaran KV. Organic matter accretion, decomposition and mineralisation. In: Nambiar EKS, Brown AG, editors, Management of soil, nutrients and water in tropical plantations forests. Canberra: ACIAR; 1997.

Santana JAS, Vilar FCR, Souto PC, Andrade LA. Acúmulo de serapilheira em plantios puros e em fragmento de mata atlântica na Floresta Nacional de Nísia - Floresta RN. Caatinga 2009; 22(3): 59-66.

Santos AP No, Barreto PAB, Gama-Rodrigues EF, Novaes AB, Paula A. Produção de serapilheira em Floresta Estacional Semidecidual e em plantios de Pterogyne nitens Tul. e Eucalyptus urophylla S. T. Blake no Sudoeste da Bahia. Ciência Florestal 2015; 25(3): 631-641. http:// dx.doi.org/10.5902/1980509819614.

Santos HG, Jacomine PKT, Anjos LHC, Oliveira VA, Oliveira JB, Coelho MR et al. Sistema brasileiro de classificação de solos. 2. ed. Rio de Janeiro: Embrapa Solos, 2006.

Santos MJC, Nascimento AVS, Mauro RA. Germinação do amendoim bravo (Pterogyne nitens Tul) para utilização na recuperação de áreas degradadas. Revista Brasileira de Ciências Agrárias 2008; 3(1): 31-34. http://dx.doi. org/10.5039/agraria.v3ila182.

Schumacher MV, Brun EJ, Rodrigues LM, Santos EM. Retorno de nutrientes via deposição de serapilheira em um povoamento de acácia-negra (Acacia mearnsii De Wild.) no Estado do Rio Grande do Sul. Revista Árvore 2003; 27(6): 791-798.

Serviço Florestal Brasileiro - SFB. Produção florestal. Brasília: 2013. [citado em 2013 jul. 05]. Disponível em: 
http://www.florestal.gov.br/snif/producao-florestal/ cadeia-produtiva.

Silva CJ, Lobo FA, Bleich ME, Sanches L. Produção de serrapilheira no Cerrado e na floresta de transição Amazônia-Cerrado do Centro Oeste brasileiro. Acta Amazonica, Manaus 2007; 37(4): 543-548. http://dx.doi. org/10.1590/S0044-59672007000400009.

Silva CJ, Lobo FA, Bleich ME, Sanches L. Contribuição de folhas na formação da serapilheira e no retorno de nutrientes em floresta de transição no Norte de mato Grosso. Acta Amazonica, Manaus 2009; 39(3): 591-600. http://dx.doi.org/10.1590/S0044-59672009000300014.

Soares AO Fo. Fitogeografia e estrutura das florestas estacionais deciduais no Brasil [tese]. Feira de Santana: Universidade Estadual de Feira de Santana; 2012.

Swift MJ, Heal OW, Anderson JM. Decomposition in terrestrial ecosystems. Oxford: Blackwell; 1979. 372 p.
Tian G, Kang BT, Brussaard L. Biological effects of plant residues with contrasting chemical compositions under humid tropical conditions- decomposition and nutrient release. Soil Biology \& Biochemistry 1992; 24(10): 10511060. http://dx.doi.org/10.1016/0038-0717(92)90035-V.

Tonini H, Arco-Verde MF, Sá SPP. Dendrometria de espécies nativas em plantios homogêneos no Estado de Roraima Andiroba (Carapa guianensis Aubl), Castanha-do-Brasil (Bertholletia excelsa Bonpl.), Ipê-roxo (Tabebuia avellanedae Lorentz ex Griseb) e Jatobá (Hymenaea courbaril L.). Acta Amazonica, Manaus 2005; 35(3): 353-362.

Veloso HP, Rangel-Filho ALR, Lima JCA. Classificação da vegetação brasileira adaptada a um sistema universal. Rio de Janeiro: IBGE; 1991. 124 p.

Vital ART, Guerrini IA, Franken WK, Fonseca RCB. Produção de serapilheira e ciclagem de nutrientes de uma floresta Estacional Semidecidual em Zona Ripária. Revista Árvore 2004; 28(6): 793-800. 